\title{
Trans-Planckian signals from the breaking of local Lorentz invariance
}

\author{
Hael Collins* \\ Department of Physics, University of Massachusetts, Amherst, Massachusetts 01003, USA \\ and The Niels Bohr International Academy, The Niels Bohr Institute, 2100 Copenhagen Ø, Denmark \\ R. Holman ${ }^{+}$ \\ Department of Physics, Carnegie Mellon University, Pittsburgh, Pennsylvania 15213, USA \\ (Received 1 June 2007; revised manuscript received 12 March 2008; published 16 May 2008)
}

\begin{abstract}
This article examines how a breakdown of a locally Lorentz-invariant, pointlike description of nature at tiny space-time intervals would translate into a distinctive set of signals in the primordial power spectrum generated by inflation. We examine the leading irrelevant operators that are consistent with the spatial translations and rotations of a preferred, isotropically expanding, background. A few of the resulting corrections to the primordial power spectrum do not have the usual oscillatory factor, which is sometimes taken to be characteristic of a "trans-Planckian" signal. Perhaps more interestingly, one of these leading irrelevant operators exactly reproduces a correction to the power spectrum that occurs in effective descriptions of the state of the field responsible for inflation.
\end{abstract}

DOI: 10.1103/PhysRevD.77.105016

PACS numbers: 11.30.Cp, 04.62.+v, 98.80.Cq

\section{INTRODUCTION}

General relativity postulates that it is always possible to treat the immediate vicinity of any place and time as though it were completely free of the influence of gravity. Near any point, space and time look flat, regardless of the wider and more complicated environment in which it happens to be situated. Looking from one place and time to another, the theory tells precisely how these locally flat frames fit together so that the global effects of gravity become apparent.

To all appearances, this postulate seems to be a reasonable principle on which to build a description of our universe. From terrestrial distances to the size of the observable universe, no discrepancy with the predictions of relativity has been found. So far, the only somewhat unsettling observation is that during the last five billion years or so, the rate at which the universe is expanding appears to have begun accelerating. But whether this fact can be attributed to a failure of the theoretical ideas behind relativity or to not having correctly accounted for all of the ingredients of the universe is still unknown.

At the opposite extreme, the idea of a locally flat reference frame is also central for quantum field theory. How quantum fields propagate through space and how they interact with each other are both strongly constrained if they are assumed to transform consistently with the symmetries of flat space. This idea additionally influences how to choose an unambiguous and unique lowest energy vacuum state. And as long as these quantum interactions occur over large enough distances for the possible quantum influences of gravity to be negligible, it is safe to treat space and time as fixed.

\footnotetext{
*hael@nbi.dk

${ }^{+}$rh4a@andrew.cmu.edu
}

Continuing to still smaller distances, a significant threshold is crossed. At intervals smaller than this threshold, the standard description of gravity begins to be strongly interacting, if viewed as a quantum theory. But since gravity corresponds to the dynamics of space-time itself, at such distances it is no longer entirely self-evident that the vicinity of any point can be locally approximated by flat space.

Many of the attempts thus far to reconcile gravity with the character of a quantum theory have in some way introduced a new length scale, whose role only becomes apparent at very short intervals. This scale is typically assumed to be close to that same threshold where gravity becomes strongly interacting. In most of these approaches, space-time at these intervals no longer has the structure and the symmetry of a classical, locally flat background.

If nature is not locally flat at short distances, some of the usual assumptions about a quantum field theory break down. The class of allowed interactions could be larger, since how fields interact only needs to be consistent with a reduced set of symmetries. Moreover, what is the true vacuum state will generally not match with a standard Lorentz-invariant vacuum at these short intervals. Whether or not such signals of a breakdown of local Lorentz invariance are seen can provide important guidance as to what postulates should be imposed when attempting to incorporate general relativity into a quantum picture of the universe.

Under ordinary circumstances, what happens at such tiny intervals would largely decouple from the interactions and space-time symmetries relevant for any currently accessible experiments, but there is one important exception. If the universe underwent a stage of accelerated expansion—or inflation [1]—during an early epoch, the ordinary fluctuations of any quantum fields present would be dramatically stretched. With enough of this stretching, fluctu- 
ations that were initially tiny would quickly grow beyond the influence of any subsequent causal process-at least while this stage of accelerated expansion lasts. Once this phase has ended and the universe starts growing at a decelerating rate, an observer sees farther and farther over time and these fluctuations - until then essentially frozen into the background space-time-can again be seen and come to influence the features of the universe at ever larger scales. This mechanism for generating a pattern of primordial fluctuations in the background space-time is an essential element of inflation.

If some form of inflation did in fact occur and provided this stage of accelerated expansion lasted sufficiently long, then it should be possible to see any signals of the violation of local Lorentz invariance through characteristic distortions in the pattern of primordial fluctuations. In practice, these fluctuations are not observed directly, but instead they are seen through their influence on the other ingredients of the universe, appearing eventually among the features of the cosmic microwave background radiation [2,3] and the distribution of matter on large scales [4].

As mentioned, one constraint imposed by local Lorentz invariance is on the choice of the vacuum state for the fields present during the inflation. A fair amount of effort [5-13] has already been made to understand how particular departures from this invariance would appear in the microwave background if they arose in new structures in the vacuum state. Most of this work examined the leading effect, without considering the radiative corrections. These corrections can actually be quite subtle [14-17] when the vacuum departs dramatically from the flat-space choice at these short intervals. To treat them properly requires either imposing very stringent constraints on the behavior of the state at short distances-essentially restricting to just the adiabatic states [18]—or modifying the propagator to account correctly for the influence of the initial, nonadiabatic state [19-21]. Despite the fact that many of the previous works have considered models thatin essence-violate local, classical Lorentz invariance in one way or another, none have studied the perhaps simpler problem of determining the influence of symmetrybreaking operators in the effective Lagrangian for inflation.

This article examines how violations of classical Lorentz invariance through such irrelevant symmetrybreaking operators influence the primordial fluctuations produced by inflation. To isolate the effects of these operators from those produced by departures of the vacuum state from the standard form, we shall choose a conventional vacuum [22] throughout-one that matches with the flat-space vacuum over infinitesimal intervals. So our goal here is twofold-partially we would like to constrain whether coordinate invariance could be broken at very short distances, but we also wish to learn the extent to which the signatures predicted by choosing nonadiabatic states during inflation can be mimicked by a less radical modification of the theory.
The next section introduces a preferred frame that breaks the symmetry between the spatial and temporal directions and that is appropriate for an inflationary background [23]. In this section we also list all of the distinct leading irrelevant operators transforming consistently with this background. Section III then evaluates the effect of each of these operators on the simplest moment of the pattern of primordial fluctuations-its two-point correlator or power spectrum. In Sec. IV, we compare these effects with the comparable signals due to nonadiabatic vacuum states. In some cases we find something familiar but we also find distinctive features too. Section $\mathrm{V}$ concludes with a brief summary and discussion of our results.

\section{PRELIMINARIES}

\section{A. Geometry}

When we consider the possible dynamics of a field theory, it is usually assumed that the underlying symmetry of nature is deeper and more extensive than what is actually realized in the particular background in which it happens to be propagating. As a simple example, the complete set of invariant quadratic operators that govern the propagation of a scalar field $\varphi$ and that are invariant under a general change of coordinates is

$$
\mathcal{L}_{\mathrm{C}}=\frac{1}{2} g^{\mu \nu} \partial_{\mu} \varphi \partial_{\nu} \varphi-\frac{1}{2} \xi R \varphi^{2}-\frac{1}{2} m^{2} \varphi^{2},
$$

where $R$ is the scalar curvature associated with the metric $g_{\mu \nu}$. The field $\varphi$ corresponds to the inflaton, the field responsible for the inflationary phase, and whose fluctuations, combined with the scalar component of the fluctuations of the metric, results in the primordial perturbations in the background space-time.

Here we examine the signatures of short-distance operators that break this general coordinate invariance, though in a way that is still consistent with the geometry of the background. $\mathcal{L}_{\mathrm{C}}$ will therefore receive corrections. These new operators are characterized by whether their importance grows (relevant or marginal corrections) or diminishes (irrelevant corrections) at larger and larger distances.

Let us begin with an isotropically expanding universe, described by a Robertson-Walker metric,

$$
d s^{2}=a^{2}(\eta) \eta_{\mu \nu} d x^{\mu} d x^{\nu}=a^{2}(\eta)\left[d \eta^{2}-d \vec{x} \cdot d \vec{x}\right] .
$$

The rate at which the scale factor $a(\eta)$ changes defines a natural energy scale associated with this geometry,

$$
H(\eta)=\frac{a^{\prime}}{a^{2}}=\frac{1}{a^{2}} \frac{d a}{d \eta},
$$

the Hubble scale. Except in the case of a de Sitter $[a(\eta) \rightarrow$ $-1 /(H \eta)]$ or a Minkowski $[a(\eta) \rightarrow 1]$ space-time, this background does not have the maximal, ten-dimensional, possible set of symmetries. We shall use this metric to define a "preferred frame" for our theory, one where the space-time is organized into spatial slices orthogonal to the 
vector,

$$
n_{\mu}=(a(\eta), 0,0,0)
$$

Because spatial symmetries - translations and rotationsare preserved by this frame, we shall allow only those operators that remain invariant under this smaller set of symmetries.

By removing the components of the metric that lie along the same direction, the normal defines an induced metric for the spatial surfaces orthogonal to it,

$$
h_{\mu \nu}=g_{\mu \nu}-n_{\mu} n_{\nu}
$$

In our Robertson-Walker frame, this induced metric is flat,

$$
h_{\mu \nu} d x^{\mu} d x^{\nu}=-a^{2}(\eta) d \vec{x} \cdot d \vec{x}
$$

being only rescaled from one surface to the next through the appearance of the scale factor.

We can use each of these tensors to construct two more by projecting the derivative of $n_{\mu}$ onto the normal and the transverse directions,

$$
n^{\lambda} \nabla_{\lambda} n_{\mu}
$$

and

$$
K_{\mu \nu}=h_{\mu}{ }^{\lambda} \nabla_{\lambda} n_{\nu}
$$

This latter tensor is the standard extrinsic curvature. In the Robertson-Walker frame, the first vanishes while the second is proportional to the Hubble scale,

$$
K_{\mu \nu} d x^{\mu} d x^{\nu}=-a^{2} H d \vec{x} \cdot d \vec{x} .
$$

Together with the standard covariant tensors,

$$
g_{\mu \nu}, \nabla_{\mu}, R_{\lambda \mu \nu \sigma}, \ldots
$$

we shall use these additional objects,

$$
n_{\mu}, h_{\mu \nu}, K_{\mu \nu}
$$

to generate the corrections to the free field theory described by $\mathcal{L}_{\mathrm{C}}$.

One final ingredient we shall use is a nonanalytic operator $\mathcal{D}$ that essentially extracts a factor of the magnitude of the spatial momentum,

$$
\mathcal{D} \equiv\left(h^{\mu \nu} \nabla_{\mu} \nabla_{\nu}-K n^{\mu} \nabla_{\mu}\right)^{1 / 2}
$$

despite its somewhat complicated form, $\mathcal{D}$ becomes more familiar once we have written it in the Robertson-Walker frame,

$$
\mathcal{D}=\frac{1}{a}(-\vec{\nabla} \cdot \vec{\nabla})^{1 / 2}
$$

Thus, for example, acting with $\mathcal{D}$ on the scalar field, written in its operator expansion,

$$
\varphi(\eta, \vec{x})=\int \frac{d^{3} \vec{k}}{(2 \pi)^{3}}\left[U_{k}(\eta) e^{i \vec{k} \cdot \vec{x}} a_{\vec{k}}+U_{k}^{*}(\eta) e^{-i \vec{k} \cdot \vec{x}} a_{\vec{k}}^{\dagger}\right]
$$

yields

$$
\mathcal{D} \varphi(\eta, \vec{x})=\int \frac{d^{3} \vec{k}}{(2 \pi)^{3}} \frac{k}{a}\left[U_{k}(\eta) e^{i \vec{k} \cdot \vec{x}} a_{\vec{k}}+U_{k}^{*}(\eta) e^{-i \vec{k} \cdot \vec{x}} a_{\vec{k}}^{\dagger}\right]
$$

\section{B. Symmetry-breaking operators}

It is now only a matter of constructing all the independent operators that can be assembled from the elements just described to learn how the signals of broken covariance would appear. Most often, the focus is on the relevant or marginal operators, since they grow most prominent at low energies, where we have the most direct experience. While we shall include such terms within our catalogue of operators here, our emphasis will instead be on the leading irrelevant operators, since they are the ones that best imitate the trans-Planckian signatures that are generated by a nonadiabatic vacuum during inflation.

In a general, maximally asymmetric background, the number of distinct symmetry-breaking operators at any particular order can be quite large. In the RobertsonWalker background, however, only a few of the symmetries are broken. Its main feature is that it treats the temporal and spatial directions differently, so the operators can be largely characterized by their relative numbers of time and space derivatives. The former scale maximally as some power of $H$, the Hubble scale, while the latter scale as powers of the spatial momentum, $\vec{k}$.

The simplest class of corrections to modify the power spectrum are those that are quadratic in the field, $\varphi$, so we shall discuss operators with this structure. Starting at dimension three - the only dimension-two operator being just the mass term-we have two possibilities: the operator,

$$
\frac{1}{3} K \varphi^{2},
$$

and the nonanalytic operator,

$$
\varphi \mathcal{D} \varphi
$$

These two are the only new relevant operators for this background.

At the next order, the four independent dimension-four terms are

$$
\frac{1}{9} K^{2} \varphi^{2}, \quad \frac{1}{3} K \varphi \mathcal{D} \varphi, \quad-h^{\mu \nu} \nabla_{\mu} \varphi \nabla_{\nu} \varphi
$$

beyond the standard kinetic and conformal terms which appeared already in $\mathcal{L}_{\mathrm{C}}$. Together these five operators describe all the possible renormalizable corrections to the covariant Lagrangian, 


$$
\begin{aligned}
\mathcal{L}_{\mathrm{R}}= & \frac{1}{3} c_{1} M K \varphi^{2}+c_{2} M \varphi \mathcal{D} \varphi+\frac{1}{9} c_{3} K^{2} \varphi^{2} \\
& +\frac{1}{3} c_{4} K \varphi \mathcal{D} \varphi-c_{5} h^{\mu \nu} \nabla_{\mu} \varphi \nabla_{\nu} \varphi
\end{aligned}
$$

Here we have introduced a new mass scale $M$ associated with whatever dynamics or principle are responsible for the broken symmetry. In the Robertson-Walker frame, $\mathcal{L}_{R}$ becomes

$$
\begin{aligned}
\mathcal{L}_{\mathrm{R}}= & c_{1} M H \varphi^{2}+\frac{c_{2}}{a} M \varphi(-\vec{\nabla} \cdot \vec{\nabla})^{1 / 2} \varphi+c_{3} H^{2} \varphi^{2} \\
& +\frac{c_{4}}{a} H \varphi(-\vec{\nabla} \cdot \vec{\nabla})^{1 / 2} \varphi+\frac{c_{5}}{a^{2}} \vec{\nabla} \varphi \cdot \vec{\nabla} \varphi .
\end{aligned}
$$

Our main interest here is the set of leading-dimensionfive-irrelevant operators. All of the standard covariant terms must contain an even power of derivatives; so at this order, the only possible operators are those explicitly violating the coordinate invariance. The many ways of contracting the many indices, combined with the choices for how the derivatives act on the fields or on the background, means that the number of operators proliferates very rapidly at higher orders. But in a fairly symmetric background, such as the Robertson-Walker space-time, only a small number of these produce distinct corrections. Moreover, in an inflationary setting, the Hubble scale typically changes only slowly, $H^{2} \gg H^{\prime}$, so among the terms where derivatives act on the background, those scaling as $H$ to some power produces the dominant effects.

Based upon these observations, we select four of the dimension-five operators that are quadratic in the field and that essentially capture all of distinctive scalings possible,

$$
\begin{aligned}
\mathcal{L}_{\mathrm{NR}}= & \frac{d_{1}}{27 M} K^{3} \varphi^{2}+\frac{d_{2}}{9 M} K^{2} \varphi \mathcal{D} \varphi-\frac{d_{3}}{3 M} K h^{\mu \nu} \nabla_{\mu} \varphi \nabla_{\nu} \varphi \\
& +\frac{d_{4}}{M} \varphi \mathcal{D}^{3} \varphi,
\end{aligned}
$$

which reduces to

$$
\begin{aligned}
\mathcal{L}_{\mathrm{NR}}= & \frac{d_{1}}{M} H^{3} \varphi^{2}+\frac{d_{2}}{a M} H^{2} \varphi(-\vec{\nabla} \cdot \vec{\nabla})^{1 / 2} \varphi \\
& +\frac{d_{3}}{a^{2} M} H \vec{\nabla} \varphi \cdot \vec{\nabla} \varphi+\frac{d_{4}}{a^{3} M} \varphi(-\vec{\nabla} \cdot \vec{\nabla})^{3 / 2} \varphi,
\end{aligned}
$$

in the Robertson-Walker frame.

\section{TRANS-PLANCKIAN CORRECTIONS TO THE POWER SPECTRUM}

The symmetry-breaking terms can have a small effect on the pattern of primordial fluctuations which, in turn, influences the cosmic microwave background and the formation of structures in the universe. To extract the basic signals of these effects, we calculate the corrections to the power spectrum of the scalar field due to the nonrenormalizable terms, the leading representative set of which composes $\mathcal{L}_{\mathrm{NR}}$. We shall work in the de Sitter limit, which is the simplest to treat analytically, although our operators are not in fact invariant under all of the generators of the symmetry group of de Sitter space.

The pattern of primordial perturbations imprinted on the background can be characterized by how fluctuations at different places are correlated with each other. For the scalar fluctuations described here, such correlations are captured by the expectation value of some number of fields each evaluated at an arbitrary position,

$$
\left\langle 0(\eta)\left|\varphi\left(\eta, \vec{x}_{1}\right) \varphi\left(\eta, \vec{x}_{2}\right) \cdots \varphi\left(\eta, \vec{x}_{n}\right)\right| 0(\eta)\right\rangle .
$$

The perturbations associated with the field $\varphi$ are initially rather small so in practice it is easier to detect the lowest order correlation functions, the simplest of which is the two-point function,

$$
\langle 0(\eta)|\varphi(\eta, \vec{x}) \varphi(\eta, \vec{y})| 0(\eta)\rangle .
$$

The two-point function is often expressed in terms of its Fourier transform, the power spectrum $P_{k}(\eta)$,

$\langle 0(\eta)|\varphi(\eta, \vec{x}) \varphi(\eta, \vec{y})| 0(\eta)\rangle=\int \frac{d^{3} \vec{k}}{(2 \pi)^{3}} e^{i \vec{k} \cdot(\vec{x}-\vec{y})}\left[\frac{2 \pi^{2}}{k^{3}} P_{k}(\eta)\right]$.

In writing this matrix element thus-with some time dependence in the state-we are implicitly working in the interaction picture. There, the evolution of the field is generated by the free Lagrangian, $\mathcal{L}_{\mathrm{C}}$, and that of the state is generated by the interacting parts, here $\mathcal{L}_{\mathrm{NR}}$.

Our purpose is to extract the general signals in the power spectrum generated by symmetry-breaking operators. We are especially interested in the extent to which these signals resemble some of the previously studied signatures of trans-Planckian physics. Such effects usually arise when the inflaton is in some nonadiabatic state; so to keep from confusing or mixing with these effects, here we shall evaluate the role of the symmetry-breaking operators in the standard vacuum, the Bunch-Davies state, which we define later in this section.

Once we have allowed the usual space-time symmetries to be broken at short distances, we have the possibility for dimension-five operators. They yield corrections to the power spectrum nominally suppressed by $H / M$. However, $H$ is not the only scale available; spatial derivatives are now possible and they appear in the form of $k \eta_{0}-$ and $k \eta$-where $\eta_{0}$ is the conformal time at which we start the evolution of the two-point function. In this respect, the symmetry-breaking operators more closely resemble some of the previous effective theory approaches [19-21] to the trans-Planckian problem, since they too produce corrections proportional to powers of $k \eta_{0}$.

Effects that depend nontrivially on the initial time $\eta_{0}$ might seem a little unfamiliar at first; so before looking at particular signatures, we should explain their meaning and describe how they restrict our theory. While such effects might seem odd, they are nothing more than an especially 
concrete manifestation of the trans-Planckian problem of inflation.

Their physical meaning becomes clearer when we introduce a scale $k_{*}$ associated with the wave number that is exactly equal to the scale $M$ at the "initial" time,

$$
\frac{k_{*}}{\left|a\left(\eta_{0}\right)\right|} \equiv M \text {. }
$$

Although we have referred to $\eta_{0}$ as an initial time, because it is when we start the evolution of the matrix elements, it does not necessarily correspond to the actual beginning of inflation. When quantum field theory is applied to scattering processes for particle experiments, we usually think in terms of asymptotic states of free particles, beginning in the far past; but we do not know whether such a picture with asymptotically free states makes sense in an inflationary background, so we are not generally guaranteed that we can take $\eta_{0}$ to the infinitely far past, $\eta_{0} \rightarrow-\infty$.

Therefore, our perspective here will be that our description of the universe only begins at $\eta_{0}$ in the Bunch-Davies state, without any assumptions about what preceded italthough some of that earlier era is presumably encoded in the symmetry-breaking operators that govern its subsequent evolution. Written in terms of $k_{*}$, we shall find that the $(H / M)\left(k \eta_{0}\right)$ corrections to the power spectrum are proportional to $k / k_{*}$. Such effects mean that our description is only applicable for scales with $k<k_{*}$.

Note that only certain classes of operators, those with larger numbers of spatial derivatives, produce these $k / k_{*}$ effects. Most others are proportional to $H / M$ and remain small in the $k \eta_{0} \rightarrow-\infty$ limit. Even the presence of effects scaling as $k / k_{*}$ does not imply that the power spectrum diverges at short wavelengths, but rather that we have left the perturbative regime for this framework. Beyond $k / k_{*}$, the total correction from all the symmetry-breaking effects can still be small. Some of the noneffective theory approaches to the trans-Planckian problem represent nonperturbative effects from this perspective-so these different pictures give useful and complementary ways of viewing the trans-Planckian problem.

\section{A. de Sitter space}

As mentioned, we evaluate the corrections to the power spectrum in the limit of a pure de Sitter space-time. In de Sitter space, the energy density of the vacuum remains constant and so the rate of expansion is also a constant, $H(\eta) \rightarrow H$. de Sitter space is also one of the three maximally symmetric space-times, so the operators that we introduced in the previous section explicitly break some of the symmetries of the background. The scale factor in this case becomes

$$
a(\eta)=-\frac{1}{H \eta},
$$

which is chosen so to agree with our previous stated con- vention that $\eta \rightarrow-\infty$ indicates the far past; the infinitely far future corresponds then to $\eta \rightarrow 0$.

Since the energy density is everywhere constant, the curvature of de Sitter space is constant too, $R=12 H^{2}$, so that there is no real distinction between the mass term and the conformal-coupling term in the free Lagrangian, $\mathcal{L}_{\mathrm{C}}$, and so we set $\xi=0$. A free field in this background then satisfies a simple Klein-Gordon equation,

$$
\left[\frac{\partial^{2}}{\partial \eta^{2}}-\frac{2}{\eta} \frac{\partial}{\partial \eta}-\vec{\nabla} \cdot \vec{\nabla}+\frac{1}{\eta^{2}} \frac{m^{2}}{H^{2}}\right] \varphi=0,
$$

which correspondingly implies a differential equation for the mode functions,

$$
U_{k}^{\prime \prime}-\frac{2}{\eta} U_{k}^{\prime}+\left[k^{2}+\frac{1}{\eta^{2}} \frac{m^{2}}{H^{2}}\right] U_{k}=0
$$

where the $U_{k}(\eta)$ are the eigenmodes associated with the operator expansion of the field,

$$
\varphi(\eta, \vec{x})=\int \frac{d^{3} \vec{k}}{(2 \pi)^{3}}\left[U_{k}(\eta) e^{i \vec{k} \cdot \vec{x}} a_{\vec{k}}+U_{k}^{*}(\eta) e^{-i \vec{k} \cdot \vec{x}} a_{\vec{k}}^{\dagger}\right]
$$

If we rescale the mode functions with a suitable factor of the conformal time, $U_{k}(\eta)=\eta^{3 / 2} Z_{\nu}(k \eta)$, and define a dimensionless variable $z=k \eta$, then the Klein-Gordon equation for the modes assumes the form of Bessel's equation,

$$
\frac{d^{2} Z_{\nu}}{d z^{2}}+\frac{1}{z} \frac{d Z_{\nu}}{d z}+\left(1-\frac{\nu^{2}}{z^{2}}\right) Z_{\nu}=0
$$

where

$$
\nu=\sqrt{\frac{9}{4}-\frac{m^{2}}{H^{2}}}
$$

The normalization of the mode $U_{k}(\eta)$ is entirely fixed by the equal time commutation relation between the field $\varphi$ and its conjugate momentum, but the second constant of integration is determined by the choice of the state. The


matches with the form of the Minkowski vacuum at short distances and is functionally

$$
U_{k}(\eta)=\frac{\sqrt{\pi}}{2} H \eta^{3 / 2} H_{\nu}^{(2)}(k \eta),
$$

where the $H_{\nu}^{(2)}(k \eta)$ is a Hankel function of the second type.

In an inflating universe, we can make one final simplifying approximation since the effective mass of the scalar field must be quite small compared with the Hubble scale, $m \ll H$. Therefore we calculate the power spectrum in the limit of a massless field, where $\nu=\frac{3}{2}$; the only danger in doing so is that the strictly massless theory can introduce infrared divergences which are an artifact of setting $m \rightarrow 0$ and which can be removed by taking a small but finite 
value for the mass of the scalar field. In the massless limit, the Bunch-Davies mode functions simplify yet further to

$$
U_{k}(\eta)=\frac{H}{k \sqrt{2 k}}(i-k \eta) e^{-i k \eta} .
$$

To have a point of comparison for the corrections from the symmetry-breaking operators, let us calculate the power spectrum of this simplest of settings,

$$
P_{k}(\eta)=\frac{k^{3}}{2 \pi^{2}} U_{k}(\eta) U_{k}^{*}(\eta)=\frac{H^{2}}{4 \pi^{2}}\left(1+k^{2} \eta^{2}\right) .
$$

The physically interesting modes-those that have been stretched well outside the horizon during inflation to become a sort of noise frozen into the background spacetime-correspond to those where $k \eta \rightarrow 0$. For these modes the power spectrum is essentially flat.

\section{B. Corrections}

Although the symmetry-breaking terms are also quadratic in the field, we shall assume that their effect is small so that they can be treated as perturbations. Since we have no knowledge of how long a stage of inflationary expansion might have lasted or what might have preceded it, ${ }^{1}$ we apply the Schwinger-Keldysh [25] approach for evaluating the corrections to the two-point function. ${ }^{2}$ The SchwingerKeldysh formalism evolves both the state $|0\rangle$ and its dual $\langle 0|$ from an initial configuration at $\eta_{0}$ to an arbitrary later time $\eta$,

$$
\langle 0(\eta)|\varphi(\eta, \vec{x}) \varphi(\eta, \vec{y})| 0(\eta)\rangle,
$$

where the time evolution of the state is given in the interaction picture by

$$
|0(\eta)\rangle=T e^{-i \int_{\eta_{0}}^{\eta} d \eta^{\prime} H_{I}\left(\eta^{\prime}\right)}|0\rangle .
$$

Here we have written the initial state more succinctly as $\left|0\left(\eta_{0}\right)\right\rangle=|0\rangle . H_{I}$ is the interaction Hamiltonian, which is, considering only the irrelevant symmetry-breaking operators,

$$
H_{I}(\eta)=-\int d^{3} \vec{x} \sqrt{-g} \mathcal{L}_{\mathrm{NR}}
$$

Before evaluating the power spectrum to first order in the corrections, we should first compare the initial and final times used for the time evolution of the state with the modes that are important for the later cosmology. For these modes, $k$ is very small compared with the conformal time by the end of inflation, $k \eta \rightarrow 0$. Therefore, we shall neglect terms that vanish in this limit. Since this is an inherently long-distance limit, we shall occasionally meet with mild

\footnotetext{
${ }^{1}$ Even were we to assume an epoch of inflation extending arbitrarily far into the past, an $S$-matrix description-just as for a purely de Sitter background [24] — would not be appropriate.

${ }^{2} \mathrm{~A}$ description of the Schwinger-Keldysh approach as it is applied to an inflationary setting is given in [19] and in [26].
}

divergences arising because we have neglected the mass of the field, a property of the theory that also obviously persists to long distances.

Furthermore, the modes responsible for the structures we are observing today should have been well within the horizon at the beginning of inflation, $\left|k \eta_{0}\right| \gg 1$. We shall therefore often take the limit where $k \eta_{0} \rightarrow-\infty$, neglecting terms that are small in this limit. In terms of the pivot momentum $k_{*}$ that we defined earlier, in a de Sitter background it is defined through

$$
\eta_{0}=-\frac{1}{k_{*}} \frac{M}{H}
$$

As we shall see, the effects that scale with a sufficient power of the spatial momentum are especially sensitive to when the initial time is chosen.

Having established these preliminaries, we can evaluate the leading corrections to the power spectrum from the dimension-five symmetry-breaking operators, listed in $\mathcal{L}_{\mathrm{NR}}$ in Eq. (2.22), to obtain

$$
\begin{aligned}
P_{k}(\eta)= & \frac{H^{2}}{4 \pi^{2}}\left[1+k^{2} \eta^{2}-2 \frac{H}{M}\left[d_{1} I_{4}\left(k \eta, k \eta_{0}\right)\right.\right. \\
& -d_{2} I_{3}\left(k \eta, k \eta_{0}\right)-d_{3} I_{2}\left(k \eta, k \eta_{0}\right) \\
& \left.\left.-d_{4} I_{1}\left(k \eta, k \eta_{0}\right)\right]+\cdots\right],
\end{aligned}
$$

where we have treated the corrections as small effects. The function $I_{n}\left(z, z_{0}\right)$ that appears in this expression corresponds to the following dimensionless integral,

$$
\begin{aligned}
& I_{n}\left(z, z_{0}\right) \\
& \quad=\int_{z_{0}}^{z} \frac{d z^{\prime}}{z^{\prime n}}\left[\left[1-z^{2}+4 z z^{\prime}-z^{\prime 2}+z^{2} z^{\prime 2}\right] \sin \left[2\left(z-z^{\prime}\right)\right]\right. \\
& \left.\quad-2\left(z-z^{\prime}\right)\left[1+z z^{\prime}\right] \cos \left[2\left(z-z^{\prime}\right)\right]\right] .
\end{aligned}
$$

At a first glance, and as expected, all of the new corrections are suppressed by $H / M$, as is familiar from a variety of models that include some nonstandard, short-distance structure in the inflaton's state [5-13]. However, this is not the only dimensionless scale available. When we extract the asymptotic behavior of these integrals in the limits,

$$
k \eta \rightarrow 0, \quad k \eta_{0} \rightarrow-\infty
$$

we shall find that several of the corrections also depend sensitively on $k \eta_{0}$. Using these limits, we look at the four corrections one by one.

\section{The correction from $K^{3} \varphi^{2} \rightarrow H^{3} \varphi^{2}$}

The first of the corrections, which contains only time derivatives, produces a small correction to the power spectrum, 


$$
P_{k}(\eta)=\frac{H^{2}}{4 \pi^{2}}\left[1+\frac{4}{3} d_{1} \frac{H}{M}[\ln |2 k \eta|-2+\gamma]+\cdots\right] .
$$

The new terms are all accompanied by the standard small factor of $H / M$, though there are already, even in this fairly innocuous term, a few differences from more standard trans-Planckian corrections. First, the correction contains a mild logarithmic divergence, $\ln |2 k \eta|$. This divergence occurs only in the long-distance, $k \eta \rightarrow 0$ limit, but its origin is quite simple to understand. In a pure de Sitter space-time, $H$ is constant so the interaction between the field and the background given by $H^{3} \varphi^{2}$ is itself essentially a mass term. If we take a very small $(m \ll H)$, but finite mass for the field, then leading contribution to the power spectrum in the $k \eta \rightarrow 0$ limit scales as

$$
P_{k}(\eta)=\frac{H^{2}}{4 \pi^{2}} \frac{4^{\nu} \Gamma^{2}(\nu)}{2 \pi}|k \eta|^{3-2 \nu}+\cdots,
$$

where $\nu$ is given in Eq. (3.10). Expanding near $\nu \sim \frac{3}{2}$ yields exactly the same structure as this "trans-Planckian" correction,

$$
P_{k}(\eta)=\frac{H^{2}}{4 \pi^{2}}\left[1+\frac{2}{3} \frac{m^{2}}{H^{2}}[\ln |2 k \eta|-2+\gamma]+\cdots\right]
$$

if we replace

$$
m^{2} \rightarrow 2 d_{1} \frac{H^{3}}{M}
$$

In the more realistic setting of a slowly rolling period of inflation, however, $H$ does contain some time dependence, so the effect of this term no longer is equivalent to that of a simple mass term.

The second difference, which appears in the next correction as well, is that the $H / M$ is not accompanied by a modulating factor, such as usually occurs in transPlanckian corrections. Very typically, imposing some cutoff or some modification in the dispersion relation of the inflaton introduces a "ringing" in the power spectrum. This "ringing" appears as an oscillatory factor, such as $\cos (2 M / H)$. The correlation between the amplitude of the correction and the frequency of the modulation is often taken as a distinctive sign of a trans-Planckian effect. Of course, such a correlation can still be taken as a distinctive signature of a state that incorporates some trans-Planckian structure, as opposed to a theory where some symmetries are explicitly broken in the Lagrangian in the transPlanckian regime. But in other instances, as we shall soon see, this clear ability to distinguish the source of a trans-Planckian effect breaks down.

\section{The correction from $K^{2} \varphi \mathcal{D} \varphi \rightarrow H^{2} \varphi(-\vec{\nabla} \cdot \vec{\nabla})^{1 / 2} \varphi$}

It might be thought that any operator that contains a factor of the spatial momentum would inevitably give corrections that diverge as $k$ grows too large. However, the correction from the next operator, $H^{2} \varphi(-\vec{\nabla} \cdot \vec{\nabla})^{1 / 2} \varphi$, shows that this fear is not realized,

$$
P_{k}(\eta)=\frac{H^{2}}{4 \pi^{2}}\left[1+d_{2} \frac{H}{M}\left[\pi+\frac{\cos \left(2 k \eta_{0}\right)}{k \eta_{0}}\right]+\cdots\right] .
$$

As with the previous correction, the observable effect of this operator is small-unless the inflationary stage is very short, so that some of the modes are near the $k \eta_{0}=-1$ limit-with only a mild scale dependence that is implicit in $H$ when we leave the ideal realm of de Sitter space and return to a slowly rolling space-time. Also, the modulating factor is again absent in the leading effect. Note that in a pure de Sitter space-time, where $H$ is constant, this correction is largely unobservable since it describes only a small rescaling.

\section{The correction from $\mathrm{Kh}^{\mu \nu} \nabla_{\mu} \varphi \nabla_{\nu} \varphi \rightarrow H \vec{\nabla} \varphi \cdot \vec{\nabla} \varphi$}

The first appearance of a direct sensitivity on the wave number occurs in the next term,

$$
P_{k}(\eta)=\frac{H^{2}}{4 \pi^{2}}\left[1+d_{3} \frac{H}{M}\left[3+\cos \left(2 k \eta_{0}\right)\right]+\cdots\right],
$$

or in terms of the threshold momentum, $k_{*}$,

$$
P_{k}(\eta)=\frac{H^{2}}{4 \pi^{2}}\left[1+d_{3} \frac{H}{M}\left[3+\cos \left(2 \frac{k}{k_{*}} \frac{M}{H}\right)\right]+\cdots\right] .
$$

In this form, the effect of the initial time is rather benign, since it only appears in the argument of the cosine factor. If the modes that we observe today were much smaller than the scale $1 / M$ at the beginning of inflation, which corresponds to $k / k_{*}$ being extremely large, such a term would introduce some fundamental noise into the power spectrum since we would not be able to resolve the frequency of the modulation, though its amplitude $(H / M)$ would still be small.

\section{The correction from $\varphi \mathcal{D}^{3} \varphi \rightarrow \varphi(-\vec{\nabla} \cdot \vec{\nabla})^{3 / 2} \varphi$}

We come now to the last and most interesting of the new corrections, that which contains the maximal number of spatial derivatives at this order. Its correction does depend sensitively on the initial time, not only through an oscillatory term, but more importantly through its amplitude,

$$
P_{k}(\eta)=\frac{H^{2}}{4 \pi^{2}}\left[1+d_{4} \frac{H}{M} k \eta_{0} \cos \left(2 k \eta_{0}\right)+\cdots\right]
$$

or equivalently

$$
P_{k}(\eta)=\frac{H^{2}}{4 \pi^{2}}\left[1-d_{4} \frac{k}{k_{*}} \cos \left(2 \frac{k}{k_{*}} \frac{M}{H}\right)+\cdots\right] .
$$


Since this correction depends linearly on the wave number $k$, it cannot be treated perturbatively once $k>k_{*}$, since all of the higher order effects become comparable to it.

If such a symmetry-breaking operator is present in the theory, then only a narrow window of modes responsible for the features that we see in the cosmic microwave background can be described perturbatively in this picture. The widest allowed range,

$$
H<\frac{k}{\left|a\left(\eta_{0}\right)\right|}<M,
$$

corresponds to when the minimal amount of inflation occurs - that is, when a fluctuation of the order of the Hubble horizon at the beginning of inflation was stretched just enough to encompass the observed universe today. The upper bound is fixed and is imposed by the requirement that the corrections to the power spectrum, coming from an operator such as $\varphi \mathcal{D}^{3} \varphi$, should remain perturbative. In de Sitter space, these bounds can also be written as

$$
\frac{H}{M}<\frac{k}{k_{*}}<1
$$

Having more than this minimal amount of inflation further constricts this range-the upper bound remains fixed, but the lower bound increases since the largest observable modes in the microwave background would have been well within the Hubble horizon even at the beginning of inflation.

We might worry that in the case of "just enough" inflation-where a fluctuation the size of the horizon at the beginning of inflation is just reentering the horizon today-some of the observable modes $k / k_{*}$ can be quite small, of the order of $H / M$. In this case we do not apply the usual assumption that $k \eta_{0} \rightarrow-\infty$. However, the largest allowed modes at the beginning of inflation can never have $\left|k \eta_{0}\right|$ smaller than 1 . Even in the limiting case, $k \eta_{0}=-1$, all of the integrals $I_{n}\left(z, z_{0}\right) \sim \mathcal{O}(1)$ (for $\left.n=1,2,3,4\right)$ up to small, order $\mathcal{O}\left(z^{2}\right)$ corrections.

\section{Higher order operators}

Among the dimension-five operators that we have analyzed, the one with the most dramatic potential signal is the operator $\varphi \mathcal{D}^{3} \varphi$. As we shall see in the next section, its signal is of the same form as one produced in an effectivestate treatment of the trans-Planckian problem. This operator is admittedly of a rather peculiar form, since it contains the nonanalytic derivative operator $\mathcal{D}$, defined in Eq. (2.12). However, none of the interesting effects that it produces are unique to $\mathcal{D}$ and we find many examples of similar effects on the power spectrum produced by higher dimensional operators.

As an example, let us consider the following dimensionsix operator,

$$
\frac{d_{5}}{M^{2}} \frac{(\vec{\nabla} \cdot \vec{\nabla} \varphi)^{2}}{a^{4}},
$$

which we have already written for a Robertson-Walker frame. Its contribution to the power spectrum, again evaluated in the $k \eta \rightarrow 0$ and $k \eta_{0} \rightarrow-\infty$ limits, is

$$
P_{k}(\eta)=\frac{H^{2}}{4 \pi^{2}}\left[1-d_{5} \frac{H^{2}}{M^{2}}\left(k \eta_{0}\right)^{2} \cos \left(2 k \eta_{0}\right)+\cdots\right]
$$

or

$$
P_{k}(\eta)=\frac{H^{2}}{4 \pi^{2}}\left[1-d_{5} \frac{k^{2}}{k_{*}^{2}} \cos \left(2 \frac{k}{k_{*}} \frac{M}{H}\right)+\cdots\right],
$$

in terms of the threshold wave number $k_{*}$.

So we see that $\left(k / k_{*}\right)^{n}$ effects are quite general and they do not depend on having defined the operator $\mathcal{D}$. The existence of these effects limits the applicability of our effective theory. Once $k \sim k_{*}$, all operators of the general form

$$
\frac{1}{M^{2 n}} \frac{1}{a^{2 n+2}}(\vec{\nabla} \cdot \vec{\nabla})^{n+1} \varphi^{2} \quad n=1,2,3, \ldots,
$$

contribute equally to the power spectrum and so the theory no longer admits a perturbative description of processes.

\section{Lower order operators}

Although our interest has been primarily in the irrelevant operators that break local Lorentz invariance, the relevant operators can produce, in principle, a much larger effect on the power spectrum and can therefore much more strongly constrain the amount of symmetry breaking that could have occurred at long distances during an inflationary era. For example, the two dimension-three operators considered earlier,

$$
\begin{aligned}
\mathcal{L}_{R} & =\frac{1}{3} c_{1} M K \varphi^{2}+c_{2} M \varphi \mathcal{D} \varphi \\
& =c_{1} M H \varphi^{2}+c_{2} \frac{M}{a} \varphi(-\vec{\nabla} \cdot \vec{\nabla})^{1 / 2} \varphi,
\end{aligned}
$$

produce the following effects in the power spectrum, again in the de Sitter limit with a massless, minimally coupled field,

$$
\begin{aligned}
P_{k}(\eta)= & \frac{H^{2}}{4 \pi^{2}}\left[1+k^{2} \eta^{2}+2 c_{1} \frac{M}{H} I_{4}\left(k \eta, k \eta_{0}\right)\right. \\
& \left.+2 c_{2} \frac{M}{H} I_{3}\left(k \eta, k \eta_{0}\right)+\cdots\right] .
\end{aligned}
$$

For the physically relevant modes $\left(k \eta \rightarrow 0\right.$ and $k \eta_{0} \rightarrow$ $-\infty)$ we find effects with essentially the same behavior as before, 


$$
\begin{aligned}
P_{k}(\eta)= & \frac{H^{2}}{4 \pi^{2}}\left[1-\frac{4}{3} c_{1} \frac{M}{H}[\ln |2 k \eta|-2+\gamma]\right. \\
& \left.+c_{2} \frac{M}{H}\left[\pi+\frac{\cos \left(2 k \eta_{0}\right)}{k \eta_{0}}\right]+\cdots\right]
\end{aligned}
$$

except that, whereas an $H / M$ suppression occurred before, here the signals are enhanced by $M / H$ and are therefore much more strongly constrained by observations.

\section{MODELS OF TRANS-PLANCKIAN SIGNALS}

By themselves, symmetry-breaking operators can provide a useful method for describing certain trans-Planckian effects; but it is interesting to learn to what extent they can be distinguished from other approaches that also produce such effects. Most approaches to the trans-Planckian problem are characterized by the fact that they assume some new principle or property of nature that only becomes important at very short distances. This principle then unambiguously fixes the state of the inflaton. What emerges in these pictures is typically not the sort of adiabatic state that is more ordinarily chosen, which is based on extrapolating the properties of nature from large scales to arbitrarily small ones.

Two broad philosophies are used for choosing these nonadiabatic states. Each has some advantages over the over, but each has its limitations as well. The first approach is to assume a specific property of nature that fixes the state. For example, we could imagine that the vacuum dispersion relation or the standard uncertainty relation become modified at distances shorter than a Planck length. Since such a property would apply continuously throughout the inflationary epoch, there is no need to fix the state at a particular initial time. However, what is learned from any one model is not generic and might have nothing to do with our universe-although with enough of these case studies the general features of a typical trans-Planckian signal do begin to emerge. Moreover, the radiative corrections in these models have not yet been thoroughly checked; whether their predictions are perturbatively stable and how they are renormalized still needs to be studied systematically.

The second approach is to apply an effective theory philosophy for including nonadiabatic structures in the state. This philosophy permits a much more general treatment and has the potential to make predictions without being restricted to any specific assumption about the shortdistance details of nature. Additionally, the loop corrections from these new structures have been studied in some detail and their divergences can be renormalized; the resulting picture forms a sensible perturbative approach. Yet, an effective theory is never meant to be a final description of nature. It is applicable up to a scale $M$; but beyond it, higher order corrections become comparable to lower order ones and the perturbative description breaks down. In an expanding background, not only does this situation impose a limit on the energies for which our description is useful, but it constrains how far back in time we can define the theory too. As we look farther back during inflation, the physical scales that we are studying will sooner or later be blue-shifted above the scale $M$ as well.

These two philosophies can also be distinguished by when or where we typically define the modes. Let us expand the inflaton $\varphi$ again,

$$
\varphi(\eta, \vec{x})=\int \frac{d^{3} \vec{k}}{(2 \pi)^{3}}\left[\varphi_{k}(\eta) e^{i \vec{k} \cdot \vec{x}} a_{\vec{k}}+\varphi_{k}^{*}(\eta) e^{-i \vec{k} \cdot \vec{x}} a_{\vec{k}}^{\dagger}\right]
$$

except that now $\varphi_{k}(\eta)$ is no longer the Bunch-Davies vacuum mode considered earlier. In quite a few models, what is done is to define a mode $\varphi_{k}$ only once it has crossed the "trans-Planckian" threshold, that is, at the time $\eta_{k}$ its physical wave number coincides the scale $M$,

$$
\frac{k}{\left|a\left(\eta_{k}\right)\right|}=M \text {. }
$$

In contrast with this picture, the effective theory approach defines all the modes simultaneously at an initial time, $\eta_{0}$, just as we did for the symmetry-breaking operators. Because of the expansion, if we are interested in some particular physical scale-for example some small feature in the cosmic microwave background, or CMB, associated with a wave number $k_{\mathrm{CMB}}$ - then we must not choose $\eta_{0}$ earlier than the time when that scale coincided with $M$,

$$
\frac{k_{\mathrm{CMB}}}{\left|a\left(\eta_{0}\right)\right|}<M \text {. }
$$

This condition is exactly the same one we encountered earlier when the theory contains operators with the maximal number of number of spatial derivatives,

$$
k_{\mathrm{CMB}}<k_{*}
$$

once we have written it in terms of $k_{*}$ rather than $\eta_{0}$.

Of course, there is a direct correspondence between a mode defined at $\eta_{k}$ as above and one of the modes on the initial time surface, $\eta_{0}$, given just by appropriately rescaling the time,

$$
k=\frac{\eta_{0}}{\eta_{k}} M
$$

The entire range of $k$ 's on the initial surface can thus be obtained by letting $\eta_{k}$ range over all possible times. So it is always possible to define the modes of both approaches along a spacelike initial surface. But we have pointed out this difference since it gives very different expectations for what is the "natural" form of trans-Planckian corrections to the power spectrum in either case. Structures defined at some $\eta_{k} \ll \eta_{0}$ when rescaled to $\eta_{0}$ will appear deeply within the trans-Planckian regime, from the vantage of a state defined along $\eta_{0}$. 
When we define the modes only as they cross a threshold, we are defining the state along a timelike surface, and the natural effects are those that vary along this surface, such as time derivatives of the scale factor, $H=a^{\prime} / a^{2}$, or time derivatives of the field. So, from this perspective, the natural trans-Planckian correction to the power spectrum tends to be suppressed by factors of $H / M$ relative to the Bunch-Davies prediction. As an illustration, when we take the de Sitter limit of [11], which uses a truncated $\alpha$ vacuum, we find just such a correction,

$$
P_{k}(\eta)=\frac{H^{2}}{4 \pi^{2}}\left\{1-2 \mathcal{O}(1) \frac{H}{M} \cos \left[2 \frac{M}{H}+\phi\right]\right\},
$$

where $\mathcal{O}(1)$ is a model-dependent, order one parameter and $\phi$ is an arbitrary phase. Now, in a pure de Sitter background, the Hubble scale $H$ is a constant so it would be nearly impossible to distinguish such a correction, which is why the first article listed in [11] calculates the correction for a more realistic, slowly rolling inflationary model. If the universe experienced a stage of power-law inflation where

$$
a(\eta)=\left(-H_{0} \eta\right)^{-(1 / 1-\epsilon)},
$$

with $\epsilon \ll 1$ being one of the dimensionless slow-roll parameters, then we can see that $H$ is no longer constant since it inherits some $k$ dependence from each mode being set at its own $\eta_{k}$.

With such a mild $k$ dependence we arrive at one of the typical predictions for how trans-Planckian physics would appear in the primordial power spectrum-as a small modulation, or a "ringing," about an otherwise flat spectrum, with an inversely related frequency and amplitude.

In contrast, the effective theory approaches contain more general corrections, some scaling as $H / M$ as before and some scaling as $k / k_{*}$, just as for the symmetry-breaking operators that we have been considering in this article. These latter corrections arise very naturally in this picture since we define the theory along an initial spacelike surface. One method $[19,20]$ for describing how a state might differ from the usual Bunch-Davies vacuum state is through a series of short-distance structures scaling as some power of $k / M$. These structures correspondingly yield corrections to the power spectrum scaling as $k / k_{*}$, and the leading correction of this form [12,21] is

$$
P_{k}(\eta)=\frac{H^{2}}{4 \pi^{2}}\left\{1+\mathcal{O}(1) \frac{k}{k_{*}} \sin \left[2 \frac{k}{k_{*}} \frac{M}{H}\right]\right\},
$$

which is exactly the same as that produced by the $\varphi \mathcal{D}^{3} \varphi$ operator.

Before comparing each of these corrections in more detail with what the symmetry-breaking operators produce, it is useful to comment on some of their features. Both exhibit a characteristic ringing-a correlation between the amplitude of the trans-Planckian modulation and its frequency. But the frequencies and the amplitudes of these two types otherwise differ quite dramatically. The $H / M$ modulation varies only slowly and presumably remains small regardless of the duration of the inflationary epoch, whereas the $k / k_{*}$ effect varies much more rapidly, especially at shorter wavelengths. The effective theory description moreover is only useful for a finite range of times and breaks down once $k \sim k_{*}$.

Most of the effects of the symmetry-breaking operators are quite different from what appeared in either of these two previous pictures. Two of the operators,

$$
H^{3} \varphi^{2}, \quad H^{2} \varphi(-\vec{\nabla} \cdot \vec{\nabla})^{1 / 2} \varphi,
$$

do not produce the ringing that is usually assumed to be the characteristic feature of a trans-Planckian signal, while the third operator,

$$
H \vec{\nabla} \varphi \cdot \vec{\nabla} \varphi,
$$

behaves like a hybrid of the earlier two approaches, with an amplitude scaling as $H / M$ but with a more rapid frequency, $k / k_{*}$.

The fourth, dimension-five, operator,

$$
\varphi(-\vec{\nabla} \cdot \vec{\nabla})^{3 / 2} \varphi
$$

however, does exactly reproduce the signal predicted by the effective initial state approaches [19-21], which perhaps should not be entirely surprising. As soon as we have included new structures in the effective state at short distances, the state itself can break the same space-time symmetries as this operator.

Yet, beneath the surface there is still an important difference between these two approaches that belies their similar signatures. For the effective-state formalism to be renormalizable, the propagator must be modified so that it remains consistent with how we have defined the effective state. For the more conventional symmetry-breaking operators we have been studying here, we have used the standard Feynman propagator and so the renormalization also proceeds more or less conventionally - with none of the boundary renormalization needed for an effective state. For example, if we consider operators that are quartic in the field such as

$$
\mathcal{L}_{\mathrm{NR}}^{(4)}=\frac{1}{36} \lambda_{1} K \varphi^{4}+\frac{1}{6} \lambda_{2} \varphi^{3} \mathcal{D} \varphi,
$$

or in the Robertson-Walker frame,

$$
\mathcal{L}_{\mathrm{NR}}^{(4)}=\frac{\lambda_{1}}{12} \frac{H}{M} \varphi^{4}+\frac{\lambda_{2}}{6} \frac{1}{a M} \varphi^{3}(-\vec{\nabla} \cdot \vec{\nabla})^{1 / 2} \varphi,
$$

these operators generate one-loop divergent corrections. The infinite parts of these corrections can then be readily removed by including counterterms of the form

$$
K \varphi^{2}, \quad \varphi \mathcal{D} \varphi .
$$

So ultimately we can regard the symmetry-breaking operators as a second, alternative effective theory descrip- 
tion. As such, it does much better at describing the signals for previous effective theory approaches to the transPlanckian problem-based either on an effective initial state $[19,20]$ or on initial boundary operators that fix the initial state [21] - than for theories where the modes are each fixed separately. From the perspective of these effective approaches, this mode-by-mode approach appears to define the state deeply in the ultraviolet regime at the initial time; such effects look nonperturbative to the effective theory and are not very readily captured. We saw this feature in the typical frequencies of the modulations. Even for different amplitudes, the operators here produced oscillating corrections with frequencies scaling as $k / k_{*}$ and not as $M / H$, though presumably such effects could be constructed with the appropriate series of higher dimensional operators.

To illustrate some of the differences between these two basic philosophies a little further, we shall examine a particular case in more detail. In particular, in Appendix A we consider how a theory with a modified dispersion relation [10] would appear from the perspective of these symmetry-breaking operators.

\section{CONCLUSIONS}

One of our goals here was to learn whether and to what extent simple symmetry-breaking operators could reproduce any of the various signatures generated by shortdistance, "trans-Planckian" structures in the state of the inflaton. These structures arise when nature is assumed to have some new physical principle-a shortest length scale, a noncommutativity, or a quantum deformation of the classical symmetries of space-time, among many other possibilities - that would cause the actual vacuum to differ substantially from the flat-space vacuum at extremely short intervals.

Although many such ideas have been applied to the vacuum state, they can largely be distinguished by whether they are established on a spacelike or a timelike surface. Within the former class are the "effective-state" treatments [19-21]. One their more distinctive signatures, a correction to the power spectrum scaling as

$$
\frac{k}{k_{*}} \cos \left(2 \frac{k}{k_{*}} \frac{M}{H}\right)
$$

can be exactly reproduced by a particular symmetrybreaking operator, described in Sec. III B 4. Note that in this work, since we have assumed a standard Bunch-Davies vacuum throughout, we have not needed to modify the propagator as in the effect state approach [19-21] to keep it consistent with the trans-Planckian structures in the state. This close agreement between the predictions of these two effective approaches provides a new insight into the physical meaning of the effective states examined in $[19,20]$ since we can now see what sorts of more conventional symmetry-breaking operators are needed to produce the same effects.

The other class of vacuum states, whose structure is modified in the trans-Planckian regime, usually defines its states along a timelike surface. In practice, what happens is that new eigenmodes of a quantum field are constantly being created to replenish earlier modes which have already red-shifted to longer wavelengths. Each mode is first defined at a time $\eta_{k}$ when its wave number is equal to a cutoff scale, $M$,

$$
\frac{k}{a\left(\eta_{k}\right)}=M .
$$

Since all of the modes are defined in exactly the same way, the "ringing" frequency in these models does not depend explicitly on $k$. Instead, it depends solely on the natural time-evolution scale of the background, $H$, in addition to $M$. Therefore, the typical correction to the power spectrum of this class,

$$
\frac{H}{M} \cos \left(2 \frac{M}{H}\right)
$$

cannot be so readily mimicked by irrelevant symmetrybreaking operators. In fact, such a signal might not ever be very naturally reproduced in an effective theory. When we fix all the modes simultaneously at an initial time, such modes must be precisely chosen at all scales, including arbitrarily fine ones which lie beyond the applicability of the effective theory. Without knowing the organizing principle that sets them, from an effective perspective such modes do not seem very natural.

Most of the work so far on testing Lorentz invariance has understandably concentrated on the possible signals of symmetry-breaking effects in high energy theory experiments [27]. Since the distances accessible to an accelerator experiment are extremely large, at least in comparison to the Planck scale, the experimentally important operators are the relevant or marginal ones. Given a particular preferred frame-for example, one such as the spatially symmetric background that we studied here-it is not too difficult to determine all of the allowed operators in the standard model which are consistent with this symmetry [28].

In an inflationary setting, the irrelevant symmetrybreaking operators can also produce measurable effects, at least in principle, since the dramatically rapid expansion of the universe effectively stretches short-distance structures to extremely large scales. Although our emphasis here has been on these operators, we should note that the constraints on relevant operators, such as $K \varphi^{2}$ and $\varphi \mathcal{D} \varphi$, are even more stringent, since their relative contribution to the power spectrum scales as $M / H$. Since very general irrelevant symmetry-breaking operators, such as those mentioned at the end of Sec. IV, tend to require relevant operators for their renormalization, some fine-tuning of the 
parameters is inevitable if we are to keep small the contribution from these lower dimension operators in the renormalized theory. However, our interest is primarily to compare with models with nonadiabatic vacuum structures-and moreover inflation is already plagued with many fine-tunings-so we have not much examined the question of naturalness here.

Tests of local Lorentz invariance provide insights into the structure of space-time at the tiniest scales. Such tests continue to be important since there seems to be a basic incompatibility between the tenets of quantum field theory and those of general relativity at distances smaller than the Planck length. Because of this impasse, it is especially vital to have some experimental guidance as to which of the postulates behind these two approaches ought to be preserved when formulating a yet more fundamental, inclusive theory of nature.

\section{ACKNOWLEDGMENTS}

This work was supported in part by the DOE Grant No. DE-FG03-91-ER40682 and the National Science Foundation Grant No. PHY02-44801. We would like to thank the reviewers of this article for suggesting the case study that appears in Appendix A. Some of the revisions of this article were made with the assistance of the EU FP6 Marie Curie Research and Training Network "UniverseNet" (MRTN-CT-2006-035863), and by the Niels Bohr International Academy.

\section{APPENDIX A: THE CASE STUDY OF A MODIFIED DISPERSION RELATION}

We mentioned that there are some differences in the general form of the corrections to the power spectrum depending on how we have defined the Lorentz-violating character of the theory. These differences are perhaps best illustrated though a detailed study of a particular example. For instance, one of the ways to introduce new transPlanckian effects is by altering a field's dispersion relation at short distances, as was done in [10] which used the following modified dispersion relation,

$$
\omega_{k}^{2}(\eta)=\frac{k^{2}}{a^{2}}-\frac{\alpha}{M^{2}} \frac{k^{4}}{a^{4}}+\frac{\beta}{M^{4}} \frac{k^{6}}{a^{6}} .
$$

Here, $\alpha$ and $\beta$ are some positive constants. ${ }^{3}$

To connect with the local Lorentz-violating operators that we have been discussing in this article, we begin with the following action,

\footnotetext{
${ }^{3}$ In the notation used in [10], $2 b_{11}=\alpha / M^{2}$ and $2 b_{12}=$ $\beta / M^{4}$.
}

$$
\begin{aligned}
S= & \frac{1}{2} \int d^{4} x \sqrt{-g}\left\{g^{\mu \nu} \partial_{\mu} \varphi \partial_{\nu} \varphi\right. \\
& +\frac{\alpha}{M^{2}}\left(g^{i j} \partial_{i} \partial_{j} \varphi\right)\left(g^{k l} \partial_{k} \partial_{l} \varphi\right) \\
& \left.+\frac{\beta}{M^{4}} g^{i j}\left(g^{k l} \partial_{i} \partial_{k} \partial_{l} \varphi\right)\left(g^{m n} \partial_{j} \partial_{m} \partial_{n} \varphi\right)\right\},
\end{aligned}
$$

where the Latin indices run only over the spatial dimensions. The symmetry-breaking operators here are higher order, of dimension six and eight. In an isotropically expanding background, expressed in conformally flat coordinates as in Eq. (2.2), this action becomes

$$
\begin{aligned}
S= & \frac{1}{2} \int d^{4} x\left\{a^{2} \varphi^{\prime 2}-a^{2} \vec{\nabla} \varphi \cdot \vec{\nabla} \varphi+\frac{\alpha}{M^{2}}(\vec{\nabla} \cdot \vec{\nabla} \varphi)^{2}\right. \\
& \left.-\frac{\beta}{a^{2} M^{4}} \delta^{i j} \partial_{i}(\vec{\nabla} \cdot \vec{\nabla} \varphi) \partial_{j}(\vec{\nabla} \cdot \vec{\nabla} \varphi)\right\},
\end{aligned}
$$

where $\vec{\nabla} \cdot \vec{\nabla}=\delta^{i j} \partial_{i} \partial_{j}$ as before. Varying with respect to the field then yields an equation of motion with the desired dispersion relation,

$$
\varphi_{k}^{\prime \prime}+2 \frac{a^{\prime}}{a} \varphi_{k}^{\prime}+a^{2} \omega_{k}^{2} \varphi_{k}=0,
$$

once we have expanded the field in its eigenmodes, $\varphi_{k}$, as in Eq. (4.1).

We can next proceed in either of two ways, each of which involves its own approximation scheme. In principle, we could apply the full dispersion relation throughout inflation by solving the modes with a WKB approximation, as long as the frequency $\omega_{k}$ remains above the inflationary Hubble scale $H$. When $\omega_{k}$ dips below $H$, this approximation does not apply and we must therefore solve the modes in each interval separately - an interval being defined by whether $\omega_{k}$ is larger or smaller than $H$-and then match these solutions on the boundary. Unfortunately even for a simple dispersion relation as in Eq. (A1), it is difficult to solve for the modes, so [10] approximates its important features with a piecewise linear dispersion relation of the form,

$$
a \omega_{k}= \begin{cases}k, & k<M \\ -s_{1} k+\left(1+s_{1}\right) M, & M<k<M_{*} \\ s_{2} k+\left(1+s_{1}\right) M-\left(s_{1}+s_{2}\right) M_{*}, & k>M_{*}\end{cases}
$$

where $s_{1}, s_{2}>0$ are slope parameters. Even with such an approximation, if we would like to be able to describe the corrections to the power spectrum perturbatively, we must further assume that the time spent in the regime where $\omega_{k}$ temporarily dips below the Hubble scale is brief, which is equivalent to requiring the following dimensionless parameter to be small.

$$
\varepsilon \equiv \frac{s_{1}}{s_{2}}-1+\frac{s_{1}}{s_{2}} \frac{\left(s_{2}-s_{1}\right) M_{*}}{\sqrt{2} H-\left(1-s_{1}\right) M} \ll 1 .
$$


The resulting power spectrum, in a pure de Sitter background, expressed as a power series in this parameter $\varepsilon$ assumes a rather complicated form, ${ }^{4}$

$$
\begin{aligned}
P_{k}(\eta) & \\
= & \frac{H^{2}}{4 \pi^{2}}\left\{1+\varepsilon\left[2 \sqrt{2}+\frac{1-s_{1}}{2} \frac{M}{H}-\frac{3\left(1-s_{1}\right)^{2}}{8 \sqrt{2}} \frac{M^{2}}{H^{2}}\right]\right. \\
& \times \sin \left[\sqrt{2}-1+\left(1-s_{1}\right) \frac{M}{H} \ln \left(1-s_{1}-\sqrt{2} \frac{H}{M}\right)\right] \\
& \left.+\mathcal{O}\left(\varepsilon^{2}\right)\right\} .
\end{aligned}
$$

It does exhibit the usual oscillatory behavior, except that now the relation between the frequency and the amplitude is no longer nearly so simple as it was before.

Alternatively, if instead of starting from the modified dispersion relation directly we were to treat the new terms as the small corrections to a free, massless scalar theory,

$$
\begin{aligned}
\sqrt{-g} \mathcal{L}_{\text {disp }}= & \frac{1}{2} \frac{\alpha}{M^{2}}(\vec{\nabla} \cdot \vec{\nabla} \varphi)^{2} \\
& -\frac{1}{2} \frac{\beta}{a^{2} M^{4}} \delta^{i j} \partial_{i}(\vec{\nabla} \cdot \vec{\nabla} \varphi) \partial_{j}(\vec{\nabla} \cdot \vec{\nabla} \varphi),
\end{aligned}
$$

then, following the approach described in Sec. III, we would arrive at the following leading corrections to the power spectrum in $\alpha$ and $\beta$,

$$
\begin{aligned}
P_{k}(\eta)= & \frac{H^{2}}{4 \pi^{2}}\left[1+k^{2} \eta^{2}+\alpha \frac{H^{2}}{M^{2}} I_{0}\left(k \eta, k \eta_{0}\right)\right. \\
& \left.+\beta \frac{H^{4}}{M^{4}} I_{-2}\left(k \eta, k \eta_{0}\right)\right],
\end{aligned}
$$

in a purely de Sitter background, as well as an $\alpha^{2}\left(H^{4} / M^{4}\right)$ effect as well, which would be of the same order as the $\beta$ correction. But since both are ultimately subleading compared with the $\alpha$ correction, we shall not write it here.

At a first glance, the corrections appear to be small, being suppressed by at least $H^{2} / M^{2}$, but of course we must expand the dimensionless integrals $I_{0}$ and $I_{-2}$ in the limit where $k \eta \rightarrow 0$ and $k \eta_{0} \rightarrow-\infty$. We then find that the leading behavior, as long as $k<k_{*}$, is

$$
P_{k}(\eta)=\frac{H^{2}}{4 \pi^{2}}\left[1+\frac{\alpha}{2} \frac{k^{2}}{k_{*}^{2}} \cos \left(2 \frac{k}{k_{*}} \frac{M}{H}\right)+\cdots\right] .
$$

Comparing these two predictions for the power spectrum, in Eq. (A7) and (A10), we can immediately see that they cover quite complementary regimes. To their credit, the symmetry-breaking operators are able to apply the dispersion relation directly, without needing to appeal to any piecewise linear approximation. Moreover, this approach does not constrain the shape of the dispersion relation to limit the time spent in the $\omega_{k}<H$ trough, as

\footnotetext{
${ }^{4}$ This expression corresponds to Eq. (110) of [10], with a slight change of notation.
}

was needed for Eq. (A6). However, since the effective theory is limited to scales where $k<k_{*}$, it does not very readily capture some of the interesting features that are within the trans-Planckian regime-in particular the dip in the dispersion relation near

$$
k=\frac{a M}{\sqrt{3 \beta}}\left[\alpha+\sqrt{\alpha^{2}-3 \beta}\right]^{1 / 2} .
$$

To see the effect of this feature would require pushing back $\eta_{0}$, or correspondingly decreasing $k_{*}$, until the perturbative description breaks down and we would then need to resum the graphs associated with multiple insertions of the interactions in Eq. (A8).

\section{APPENDIX B: INTEGRALS}

This short appendix describes some of the asymptotic behavior of the dimensionless integrals that we encountered when we calculated the corrections to the power spectrum from the symmetry-breaking operators. Recall that the general form of these integrals is

$$
\begin{aligned}
& I_{n}\left(z, z_{0}\right) \\
& \quad=\int_{z_{0}}^{z} \frac{d z^{\prime}}{z^{\prime n}}\left[\left[1-z^{2}+4 z z^{\prime}-z^{\prime 2}+z^{2} z^{\prime 2}\right] \sin \left[2\left(z-z^{\prime}\right)\right]\right. \\
& \left.\quad-2\left(z-z^{\prime}\right)\left[1+z z^{\prime}\right] \cos \left[2\left(z-z^{\prime}\right)\right]\right] .
\end{aligned}
$$

The specific cases that occur for the dimension-five operators are $n=1,2,3$, and 4 . Remember also that the arguments correspond to the final and initial conformal times scaled in terms of the wave number of a mode, $z=k \eta$ and $z_{0}=k \eta_{0}$.

Inflation works by stretching a mode, which will eventually produce some observable feature of our universe, well outside of the Hubble horizon during inflation,

$$
\frac{k}{|a(\eta)|} \ll H(\eta)
$$

which for a de Sitter background becomes,

$$
k|\eta| \ll 1 \quad \text { or } \quad z=k \eta \rightarrow 0 .
$$

We shall therefore expand each of the relevant cases in this limit, 


$$
\begin{aligned}
I_{4}\left(z, z_{0}\right)= & \frac{2}{3} \operatorname{Ci}\left(2 z_{0}\right)-\frac{\left(1+z_{0}^{2}\right) \sin \left(2 z_{0}\right)-2 z_{0} \cos \left(2 z_{0}\right)}{3 z_{0}^{3}} \\
& -\frac{2}{3}[\ln |2 z|-2+\gamma]+\mathcal{O}\left(z^{2}\right) \\
I_{3}\left(z, z_{0}\right)= & \operatorname{Si}\left(2 z_{0}\right)+\frac{\cos \left(2 z_{0}\right)}{z_{0}}-\frac{1}{2} \frac{\sin \left(2 z_{0}\right)}{z_{0}^{2}}+\mathcal{O}\left(z^{2}\right) \\
I_{2}\left(z, z_{0}\right)= & \frac{3}{2}+\frac{1}{2} \cos \left(2 z_{0}\right)-\frac{\sin \left(2 z_{0}\right)}{z_{0}}+\mathcal{O}\left(z^{2}\right) \\
I_{1}\left(z, z_{0}\right)= & \frac{1}{2} z_{0} \cos \left(2 z_{0}\right)-\frac{5}{4} \sin \left(2 z_{0}\right)+\operatorname{Si}\left(2 z_{0}\right)+\mathcal{O}\left(z^{2}\right) .
\end{aligned}
$$

The only absolute limit on the initial time is that-at the very least-relevant modes should have been within the Hubble horizon at the beginning of inflation,

$$
\frac{k}{\left|a\left(\eta_{0}\right)\right|}>H\left(\eta_{0}\right)
$$

Again, in a de Sitter background this requires,

$$
z_{0}=k \eta_{0}<-1 \text {. }
$$

This bound is only saturated if inflation lasted just long enough that a mode of the size of the Hubble horizon is just reentering the horizon today. Most inflationary models produce much more expansion than this minimal amount and even in this extremal case most modes will be smaller than the Hubble horizon. Therefore, we shall usually examine the limit $z_{0} \rightarrow-\infty$. In any event, each of these integrals is of $\mathcal{O}(1)$ for $z_{0}=-1$ and $z$ small.

As we allow $\eta_{0}$ to extend arbitrarily far back in the past, we very soon encounter trans-Planckian modes-modes whose wavelength was smaller than a Planck length $1 / M_{\mathrm{pl}}$ at the beginning of inflation,

$$
\left|z_{0}\right|>\frac{M_{\mathrm{pl}}}{H} .
$$

Let us therefore expand the integrals in the limit $z_{0} \rightarrow-\infty$ to learn how sensitively they can depend on these transPlanckian modes,

$$
\begin{aligned}
& I_{4}\left(z, z_{0}\right)=-\frac{2}{3}[\ln |2 z|-2+\gamma]+\mathcal{O}\left(z^{2}, z_{0}^{-2}\right) \\
& I_{3}\left(z, z_{0}\right)=\frac{\pi}{2}+\frac{\cos \left(2 z_{0}\right)}{2 z_{0}}+\mathcal{O}\left(z^{2}, z_{0}^{-2}\right) \\
& I_{2}\left(z, z_{0}\right)=\frac{3}{2}+\frac{1}{2} \cos \left(2 z_{0}\right)+\mathcal{O}\left(z^{2}, z_{0}^{-1}\right) \\
& I_{1}\left(x, x_{0}\right)=\frac{z_{0}}{2} \cos \left(2 z_{0}\right)+\frac{\pi}{2}-\frac{5}{4} \sin \left(2 z_{0}\right)+\mathcal{O}\left(z^{2}, z_{0}^{-1}\right) .
\end{aligned}
$$

Of these four cases, it is the last that depends most sensitively on trans-Planckian physics, since it scales linearly with $z_{0}=k \eta_{0}$.
[1] A. Linde, Particle Physics and Inflationary Cosmology (Harwood Academic, Chur, Switzerland, 1990); E.W. Kolb and M.S. Turner, The Early Universe (AddisonWesley, Reading, MA, 1990); A. Liddle and D. Lyth, Cosmological Inflation and Large-Scale Structure (Cambridge University Press, Cambridge, England, 2000); S. Dodelson, Modern Cosmology (Academic Press, San Diego, CA, 2003).

[2] U. Seljak, Astrophys. J. 435, L87 (1994); W. Hu and N. Sugiyama, Astrophys. J. 444, 489 (1995); U. Seljak and M. Zaldarriaga, Astrophys. J. 469, 437 (1996).

[3] D. N. Spergel et al., Astrophys. J. Suppl. Ser. 170, 377 (2007); H. V. Peiris et al., Astrophys. J. Suppl. Ser. 148, 213 (2003); D. N. Spergel et al. (WMAP Collaboration), Astrophys. J. Suppl. Ser. 148, 175 (2003); C. L. Bennett et al., Astrophys. J. Suppl. Ser. 148, 1 (2003).

[4] D. J. Eisenstein et al. (SDSS Collaboration), Astrophys. J. 633, 560 (2005).

[5] J. Martin and R.H. Brandenberger, Phys. Rev. D 63, 123501 (2001); R.H. Brandenberger and J. Martin, Mod. Phys. Lett. A 16, 999 (2001).

[6] N. Kaloper, M. Kleban, A. E. Lawrence, and S. Shenker, Phys. Rev. D 66, 123510 (2002); N. Kaloper, M. Kleban, A. Lawrence, S. Shenker, and L. Susskind, J. High Energy Phys. 11 (2002) 037.
[7] C. P. Burgess, J. M. Cline, F. Lemieux, and R. Holman, J. High Energy Phys. 02 (2003) 048; C.P. Burgess, J. M. Cline, and R. Holman, J. Cosmol. Astropart. Phys. 10 (2003) 004; C. P. Burgess, J. Cline, F. Lemieux, and R. Holman, arXiv:astro-ph/0306236.

[8] R. Easther, B. R. Greene, W. H. Kinney, and G. Shiu, Phys. Rev. D 64, 103502 (2001); 67, 063508 (2003); G. Shiu and I. Wasserman, Phys. Lett. B 536, 1 (2002); R. Easther, B. R. Greene, W. H. Kinney, and G. Shiu, Phys. Rev. D 66, 023518 (2002).

[9] J. C. Niemeyer, Phys. Rev. D 63, 123502 (2001); A. Kempf, Phys. Rev. D 63, 083514 (2001); J. C. Niemeyer and R. Parentani, Phys. Rev. D 64, 101301 (2001); A. Kempf and J.C. Niemeyer, Phys. Rev. D 64, 103501 (2001); A. Kempf and L. Lorenz, Phys. Rev. D 74, 103517 (2006); A. A. Starobinsky, Pisma Zh. Eksp. Teor. Fiz. 73, 415 (2001) [JETP Lett. 73, 371 (2001)]; L. Hui and W. H. Kinney, Phys. Rev. D 65, 103507 (2002); S. Shankaranarayanan, Classical Quantum Gravity 20, 75 (2003); S. F. Hassan and M. S. Sloth, Nucl. Phys. B674, 434 (2003); K. Goldstein and D. A. Lowe, Phys. Rev. D 67, 063502 (2003); V. Bozza, M. Giovannini, and G. Veneziano, J. Cosmol. Astropart. Phys. 05 (2003) 001; G. L. Alberghi, R. Casadio, and A. Tronconi, Phys. Lett. B 579, 1 (2004); U. H. Danielsson, Phys. Rev. D 66, 023511 
(2002); U. H. Danielsson, J. High Energy Phys. 07 (2002) 040; R. H. Brandenberger and J. Martin, Int. J. Mod. Phys. A 17, 3663 (2002); S. Cremonini, Phys. Rev. D 68, 063514 (2003).

[10] J. Martin and R. Brandenberger, Phys. Rev. D 68, 063513 (2003).

[11] J. Martin and C. Ringeval, Phys. Rev. D 69, 083515 (2004); 69, 127303 (2004); J. Cosmol. Astropart. Phys. 01 (2005) 007; J. Cosmol. Astropart. Phys. 08 (2006) 009.

[12] R. Easther, W. H. Kinney, and H. Peiris, J. Cosmol. Astropart. Phys. 05 (2005) 009; J. Cosmol. Astropart. Phys. 08 (2005) 001.

[13] D. Boyanovsky, H. J. de Vega, and N. G. Sanchez, Phys. Rev. D 74, 123006 (2006); Phys. Rev. D 74, 123007 (2006).

[14] M. B. Einhorn and F. Larsen, Phys. Rev. D 67, 024001 (2003); 68, 064002 (2003).

[15] K. Goldstein and D. A. Lowe, Nucl. Phys. B669, 325 (2003); Phys. Rev. D 69, 023507 (2004).

[16] H. Collins, R. Holman, and M. R. Martin, Phys. Rev. D 68, 124012 (2003); H. Collins and M. R. Martin, Phys. Rev. D 70, 084021 (2004); H. Collins, arXiv:hep-th/0312144.

[17] H. Collins and R. Holman, Phys. Rev. D 70, 084019 (2004); H. Collins, Phys. Rev. D 71, 024002 (2005); H. Collins, arXiv:hep-th/0410228.

[18] P. R. Anderson, C. Molina-Paris, and E. Mottola, Phys. Rev. D 72, 043515 (2005).

[19] H. Collins and R. Holman, Phys. Rev. D 71, 085009 (2005).

[20] H. Collins and R. Holman, arXiv:hep-th/0507081; Phys.
Rev. D 74, 045009 (2006); arXiv:hep-th/0609002.

[21] K. Schalm, G. Shiu, and J.P. van der Schaar, J. High Energy Phys. 04 (2004) 076; B. Greene, K. Schalm, J. P. van der Schaar, and G. Shiu, in Proceedings of the 22nd Texas Symposium on Relativistic Astrophysics at Stanford University, eConf C041213, 0001 (2004); B. R. Greene, K. Schalm, G. Shiu, and J.P. van der Schaar, J. Cosmol. Astropart. Phys. 02 (2005) 001; K. Schalm, G. Shiu, and J. P. van der Schaar, AIP Conf. Proc. 743, 362 (2005).

[22] T. S. Bunch and P.C. Davies, Proc. R. Soc. A 360, 117 (1978).

[23] Other preferred frames, such as one that breaks rotational invariance, can also be useful for trying the explain the alignment of some of the large-scale multipoles in the microwave background, as was done in L. Ackerman, S. M. Carroll, and M. B. Wise, Phys. Rev. D 75, 083502 (2007).

[24] E. Witten, arXiv:hep-th/0106109.

[25] J. S. Schwinger, J. Math. Phys. (N.Y.) 2, 407 (1961); L. V. Keldysh, Zh. Eksp. Teor. Fiz. 47, 1515 (1964) Sov. Phys. JETP 20, 1018 (1965); K. T. Mahanthappa, Phys. Rev. 126, 329 (1962); P. M. Bakshi and K. T. Mahanthappa, J. Math. Phys. (N.Y.) 4, 12 (1963).

[26] S. Weinberg, Phys. Rev. D 72, 043514 (2005); 74, 023508 (2006).

[27] See, for example, D. Colladay and V. A. Kostelecky, Phys. Rev. D 55, 6760 (1997); 58, 116002 (1998).

[28] S. R. Coleman and S.L. Glashow, Phys. Rev. D 59, 116008 (1999). 